\title{
The thermal ignition problem in a cube
}

\author{
S. D. Watt* G. N. Mercer ${ }^{\dagger} \quad$ H. S. Sidhu \\ R. O. Weber ${ }^{\S}$
}

(Received 14 January 2003)

\begin{abstract}
Steady state solutions for spontaneous thermal ignition in a unit cube are considered. For the unit sphere there are numerous solutions possible: these appear as "wiggles" in the bifurcation diagram. For the unit sphere this has been shown analytically. In contrast, for the unit cube analytic solutions are not possible and hence we must resort to numerical methods to determine the corresponding bifurcation diagram. Comparisons between the cube and the sphere are made.
\end{abstract}

*Department of Mathematics and Statistics, University of Birmingham, Edgbaston B15 2TT, UNITED KInGDOM. mailto:simon_watt@bigfoot.com

${ }^{\dagger}$ School of Mathematics and Statistics, UNSW at ADFA, Canberra ACT 2600, Australia. mailto:g.mercer@adfa.edu.au

${ }^{\ddagger}$ School of Mathematics and Statistics, UNSW at ADFA, Canberra ACT 2600, Australia. mailto:h.sidhu@adfa.edu.au

${ }^{\S}$ School of Mathematics and Statistics, UNSW at ADFA, Canberra ACT 2600, Australia. mailto:r.weber@adfa.edu.au

${ }^{0}$ See http://anziamj . austms.org.au/V44/CTAC2001/Watt for this article, (C) Austral. Mathematical Soc. 2003. Published 1 April 2003. ISSN 1446-8735 


\section{Contents}

1 Introduction

C821

2 The thermal ignition problem in a sphere

C822

3 Mathematical model of thermal ignition in a cube $\mathbf{C 8 2 2}$

4 Numerical method

C824

5 Turning the corner

C830

6 Conclusions

C834

References

C834

\section{Introduction}

We consider the thermal ignition problem in a cube. This problem is well studied in various geometries, in particular in the sphere [1, $2,4,5]$. One of the interesting properties of ignition in the sphere is that there can be a very large number of solutions to the steadystate problem. The sphere problem can be studied both numerically and, to some extent, analytically. However, in the cube, ignition can really only be attacked numerically and for this case we chose to solve the problem using a finite difference approximation to the steady-state equations. Then using a relaxation iterative method, we find the "cold" branch of the bifurcation diagram. From this, we find what seems to be an almost linear connection between the behaviour in the sphere and cube. Using an averaging method, we extend the numerical exploration to find the first few branches of the bifurcation diagram. Again, an almost linear connection between 
the cube and sphere is found.

\section{The thermal ignition problem in a sphere}

First, let us revisit the steady-state thermal ignition problem in a unit sphere. As in [5], we assume the temperature profile is axisymmetric, then the problem to be solved is the ODE

$$
u^{\prime \prime}(r)+\frac{2}{r} u^{\prime}(r)+\delta e^{u(r)}=0,
$$

with $u^{\prime}(0)=0$ and $u(1)=0$. This problem can easily be solved numerically and the bifurcation diagram is drawn in Figure 1. See that for a range of the parameter $\delta$, there are multiple solutions to the problem. For $\delta=2$, there are an infinite number of solutions.

\section{Mathematical model of thermal ignition in a cube}

Consider the evolution of a temperature profile in a cube of side length one, where the faces of the cube are kept at ambient temperature. This can be modelled by a heat balance equation

$$
\rho c_{p} \frac{\partial T}{\partial t}=\kappa \nabla^{2} T+Q A e^{-E / R T},
$$

with boundary conditions $T(0, y, z)=T(1, y, z)=T(x, 0, z)=$ $T(x, 1, z)=T(x, y, 0)=T(x, y, 1)=T_{a}$; and where $\rho$ is the density, 


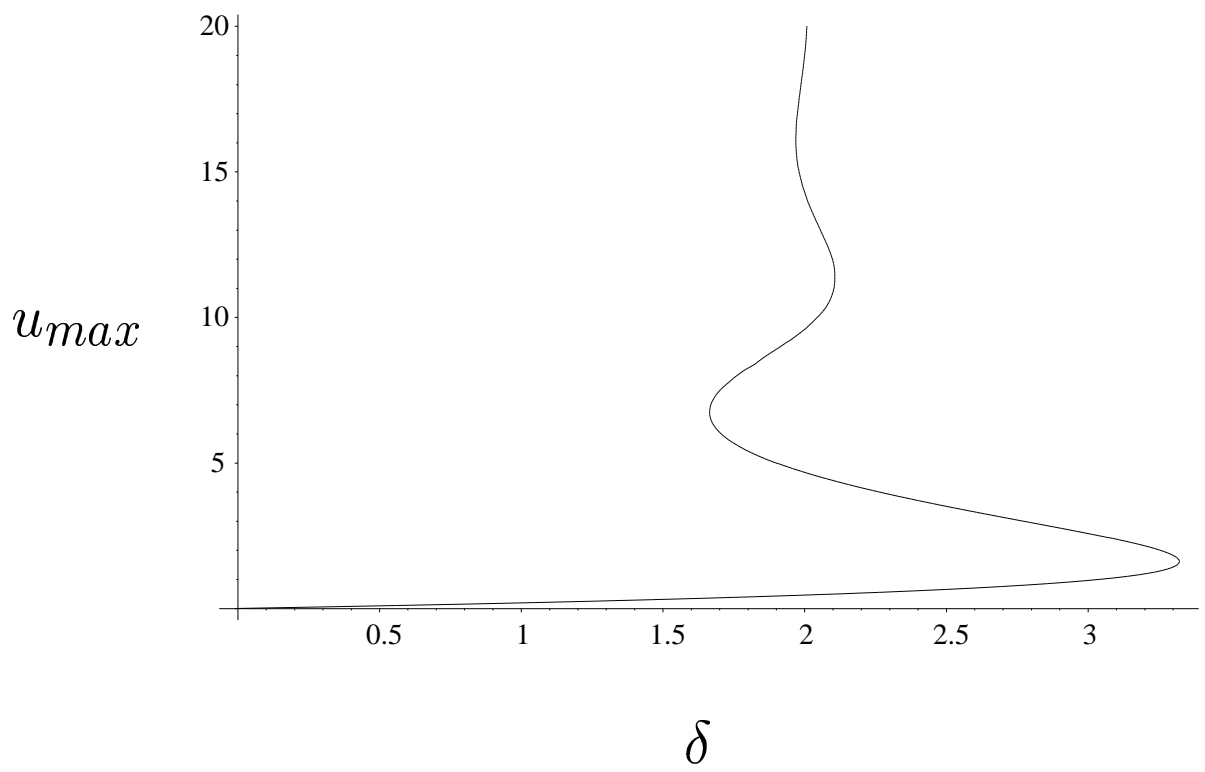

Figure 1: Bifurcation diagram showing the multiple solutions to the steady-state problem in a sphere, where $u_{\max }$ is the maximum temperature in the sphere. 
$c_{p}$ is specific heat, $T$ is the temperature, $\kappa$ is the thermal conductivity, $Q$ is the latent heat, $A$ is the Arrhenius pre-exponential, $E$ is the activation energy and $R$ is the universal gas constant.

Non-dimensionalising time, space and temperature, as described in [2], this problem is rewritten as

$$
\frac{\partial u}{\partial t}=\nabla^{2} u+\delta e^{u /(1+\epsilon u)},
$$

with $u(0, y, z)=u(1, y, z)=u(x, 0, z)=u(x, 1, z)=u(x, y, 0)=$ $u(x, y, 1)=0$.

We now consider the steady problem

$$
\nabla^{2} u+\delta e^{u}=0
$$

where we have also set $\epsilon=0$ as it is a small number for most physically realistic substances.

\section{Numerical method}

Unlike the sphere, the cube problem cannot be reduced to an ODE. The only method of solution is numerical. The method we choose is a relaxation iterative method [3], using a finite difference approximation to the derivatives. Due to the symmetry of the problem, we need only look at one eighth of the cube. (We can in fact reduce the problem by another factor of eight by considering the diagonal symmetries, but for clarity of presentation we are content with a one eighth cube.)

The steady-state problem reduces to the non-linear equation for each grid point

$$
u_{i+1, j, k}+u_{i-1, j, k}+u_{i, j+1, k}+u_{i, j-1, k}+u_{i, j, k+1}+u_{i, j, k-1}
$$




$$
-6 u_{i, j, k}+\delta \Delta^{2} e^{u_{i, j, k}}=0,
$$

where $\Delta$ is the grid spacing, and $i, j$ and $k$ vary over the $N$ grid points in each direction.

Solving this equation for $u_{i, j, k}$ and using updated values (this method is the Gauss-Seidel method [3]) has order $N^{3}$ convergence. However, using a relaxation method, the convergence can be sped up to be almost linear.

At each grid point, one performs the calculation of a residual

$$
\begin{aligned}
\xi_{i, j, k}= & u_{i+1, j, k}+u_{i-1, j, k}+u_{i, j+1, k}+u_{i, j-1, k}+u_{i, j, k+1}+u_{i, j, k-1} \\
& -6 u_{i, j, k}+\delta \Delta^{2} e^{u_{i, j, k}}
\end{aligned}
$$

and then iterates using the relation

$$
u_{i, j, k}^{\text {updated }}=u_{i, j, k}+\omega \frac{\xi_{i, j, k}}{6} .
$$

This process is repeated until the solution is within some set tolerance.

This method will converge for $0<\omega<2$ : for $0<\omega<1$ the method is "under-relaxed"; for $\omega=1$ the method is equivalent to the Gauss-Seidel method; and for $1<\omega<2$ the method is "over-relaxed". For every problem, the optimal relaxation parameter needs to be found. As shown in Figure 2, the optimal relaxation parameter for the current problem is about 1.9.

Using this method, we find the "cold" or stable solution to the problem in a cube, shown in Figure 3. Also shown are the curves for the smallest sphere that bounds the cube (radius $\sqrt{3} / 2$ ) and the largest sphere which fits inside the cube (radius 1/2). As expected physically the cube solution lies between its bounding sphere solutions. 


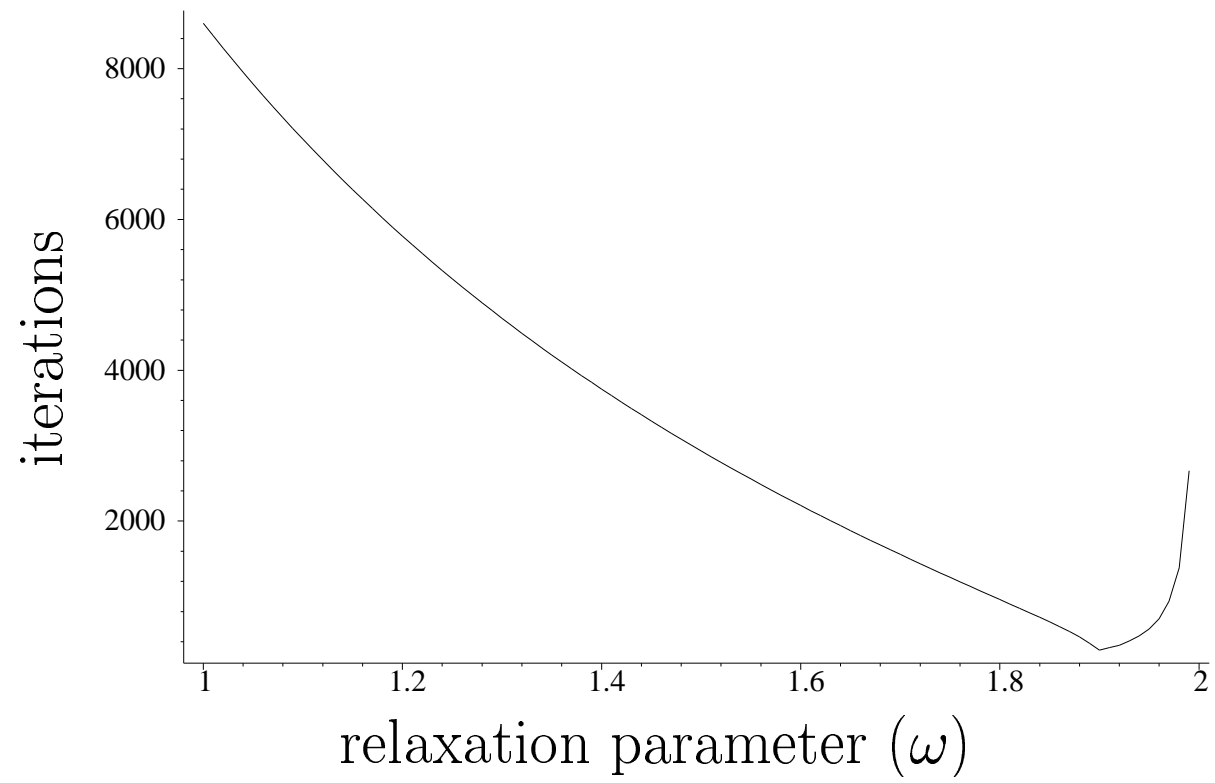

FiguRE 2: Graph of the number of iteration needed for convergence in the cube problem, for various relaxation parameters $\omega$. 


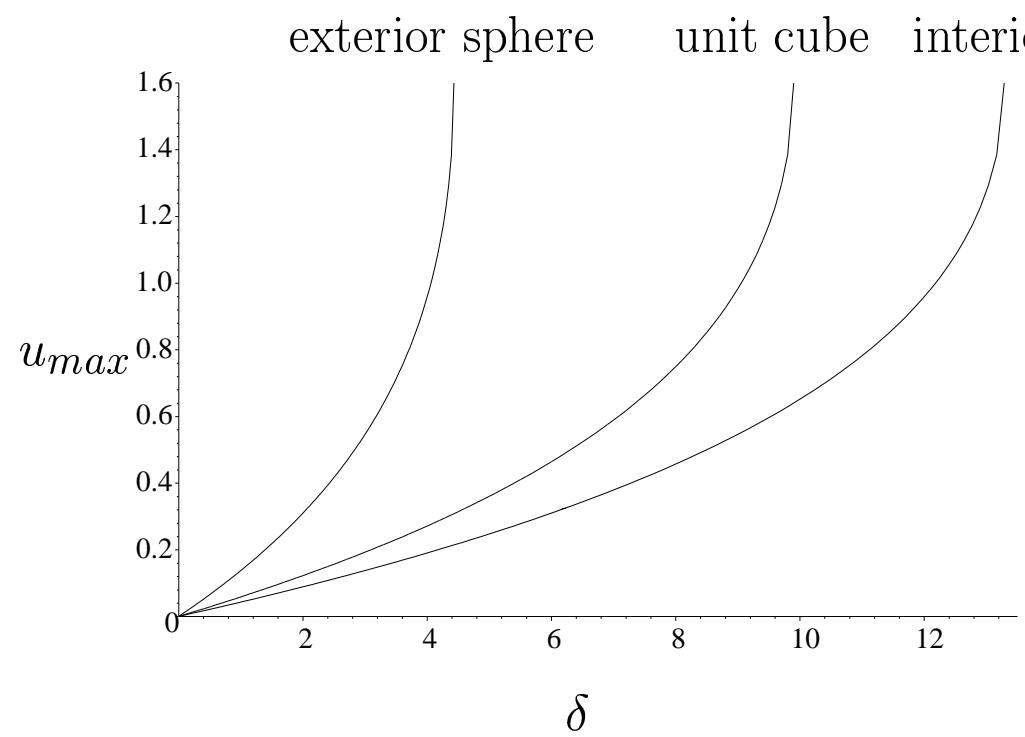

FiguRE 3: Graph of the stable branch for the cube, along with the internal and exterior bounding spheres. 


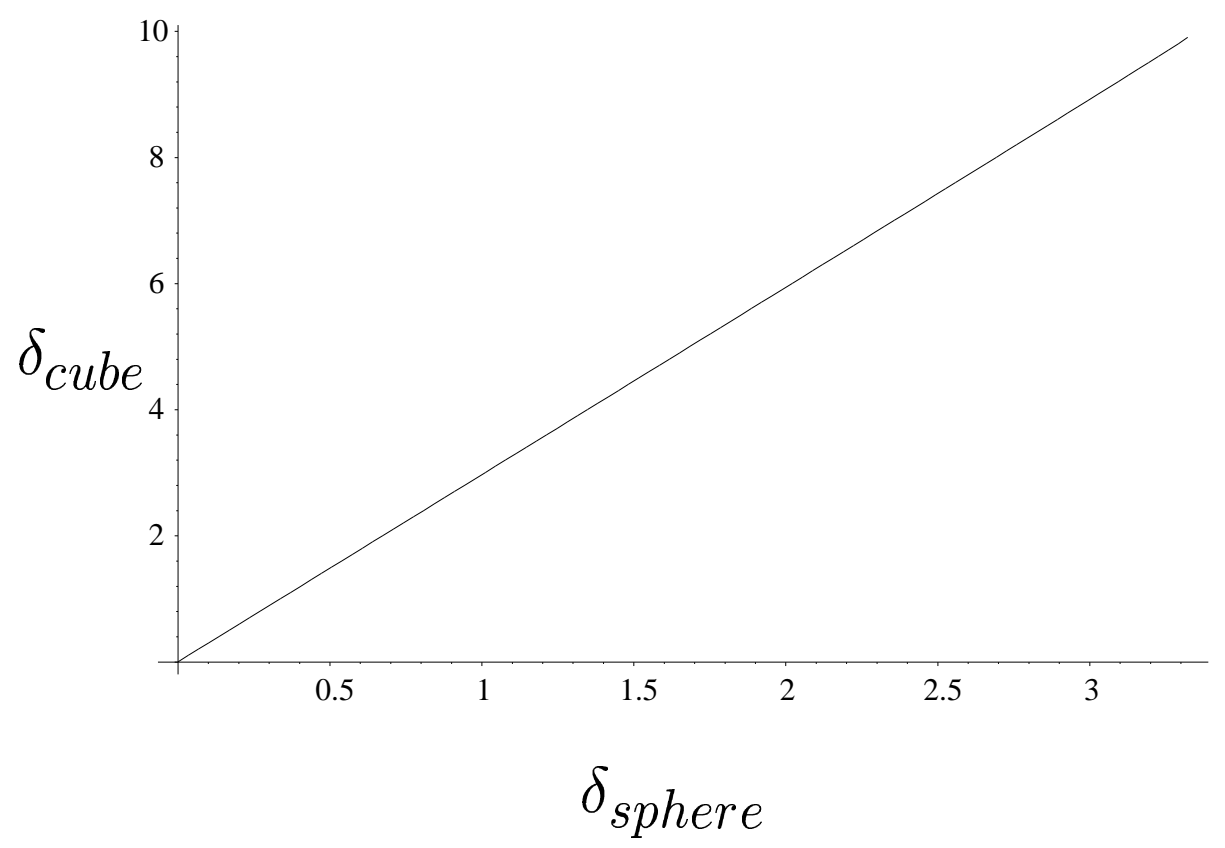

Figure 4: Comparison of the parameter values in the cube and the sphere, parameterised by the maximum temperature.

As another comparison between the cube and sphere, look in Figure 4 at the solutions, parametrised by the maximum temperature, which occur in the centre of the cube and sphere respectively: there is a linear relationship between the $\delta$ in the cube and the sphere.

As a rather ad hoc justification to the connection between the sphere and the cube, we look at the temperature profile, or more correctly, the distributions of radii from the centre of the cube for different surfaces of constant temperature. See in Figure 5 that the profile is seemingly spherical near the centre, where there is little variation in radii, but then loses this property as the temperature reduces and the edge effects start to dominate. 


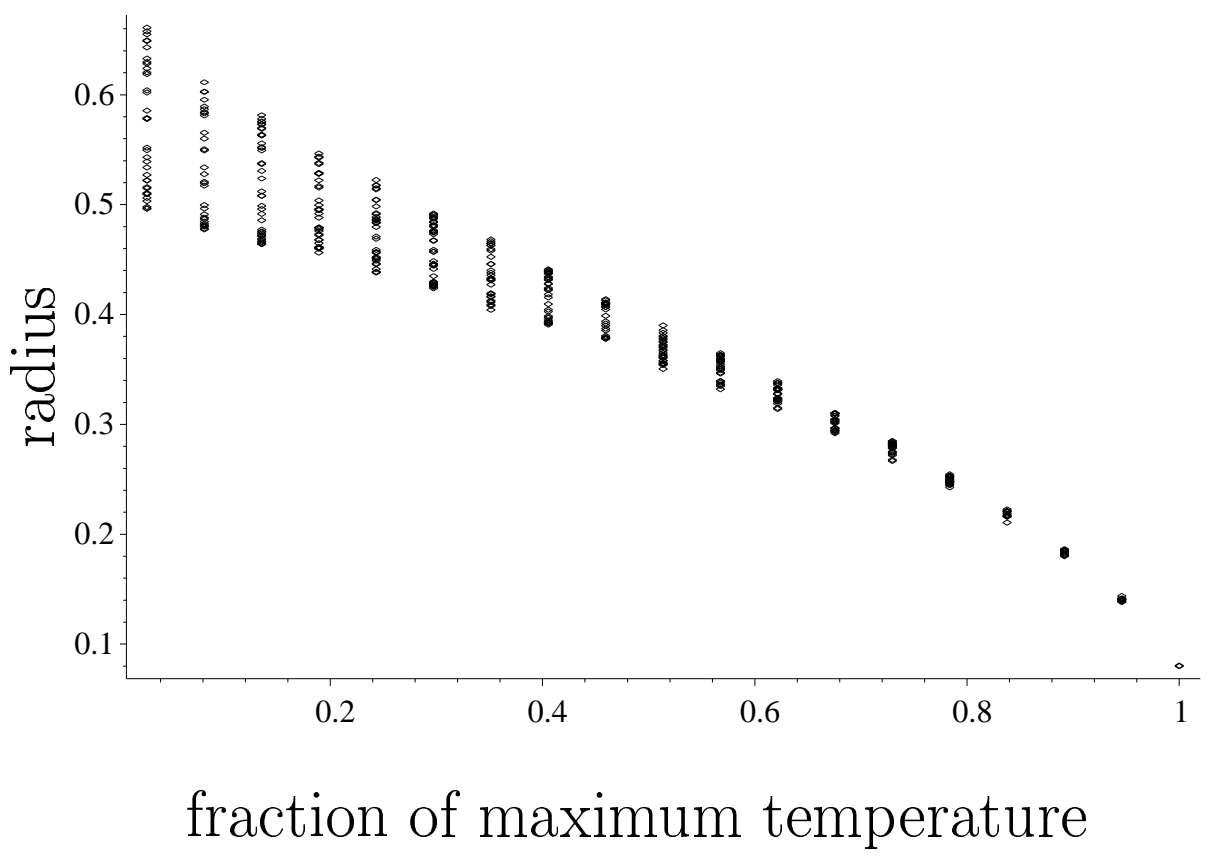

FiguRE 5: Graph of the radii from the centre for various surfaces of constant temperature. 


\section{Turning the corner}

In the previous section, we found the stable branch. In the sphere, the bifurcation diagram can be found by inverting the problem: that is, by specifying the maximum temperature, and then the corresponding parameter value $\delta$ is determined. We would like to be able to do the same thing in the cube. By using an averaging method, this turns out to be nearly possible and more of the bifurcation diagram is determined, as described below.

Consider the finite difference equations we need to solve, written as

$$
\tilde{\nabla}^{2} u_{i, j, k}+\delta e^{u_{i, j, k}}=0,
$$

where $\tilde{\nabla}^{2}$ is the finite difference approximation to $\nabla^{2}$.

If we now multiply each equation by some weighting function and sum over all grid points, we can then solve for $\delta$

$$
\delta=-\frac{\sum_{i, j, k} w_{i, j, k} \tilde{\nabla}^{2} u_{i, j, k}}{\sum_{i, j, k} w_{i, j, k} e^{u_{i, j, k}}} .
$$

The solution method is a combination of relaxation, as described in a previous section, resetting the maximum temperature at the centre as prescribed and then carrying out of the averaging step.

For $w_{i, j, k}=1$, this method converges for maximum non-dimensionalised temperatures up to approximately 5.0, which is about halfway along the first unstable branch. For

$$
w_{i, j, k}=\exp \left\{-0.01\left[(i-h)^{2}+(j-h)^{2}+(k-h)^{2}\right]\right\},
$$

where $h=N / 2$ (half the number of grid points in each direction), this method converges for maximum temperatures up to about 8.0, which is somewhere along the second unstable branch. 


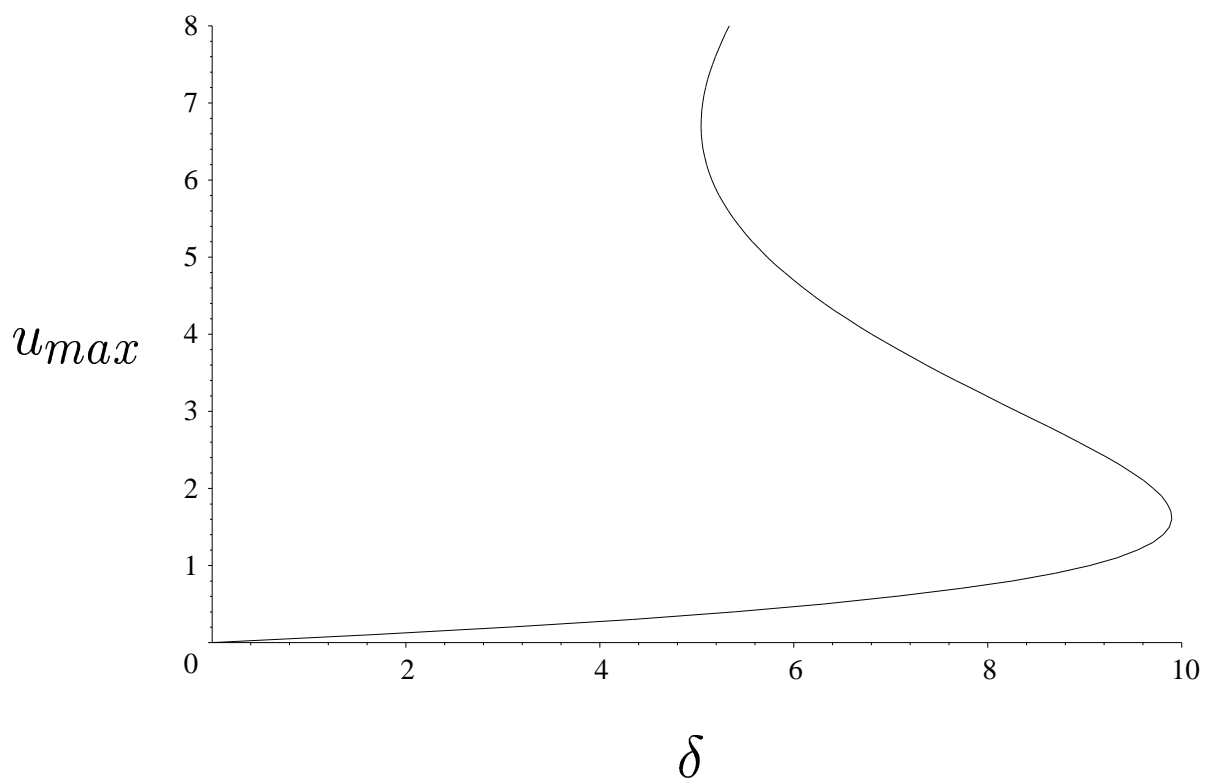

FiguRE 6: Bifurcation diagram showing the multiple solutions to the steady-state problem in a cube

In Figure 6, see a resemblance with the sphere (cf. Figure 1) and in Figure 7, see that there is still an almost linear relationship between the steady-state solutions in the cube and sphere.

As a final comparison between the cube and the sphere, in Figure 8 , we have plotted the derivatives of the corresponding parameter values, $\delta$, in the cube and the sphere with respect to the maximum temperature. See that the turning point, where the derivatives vanish, nearly coincide in both cases. This suggests a fundamental connection between the thermal ignition problem in the cube and the sphere, probably related to our earlier observation about the almost spherical nature of the temperature iso-surfaces in the central region of the cube. 


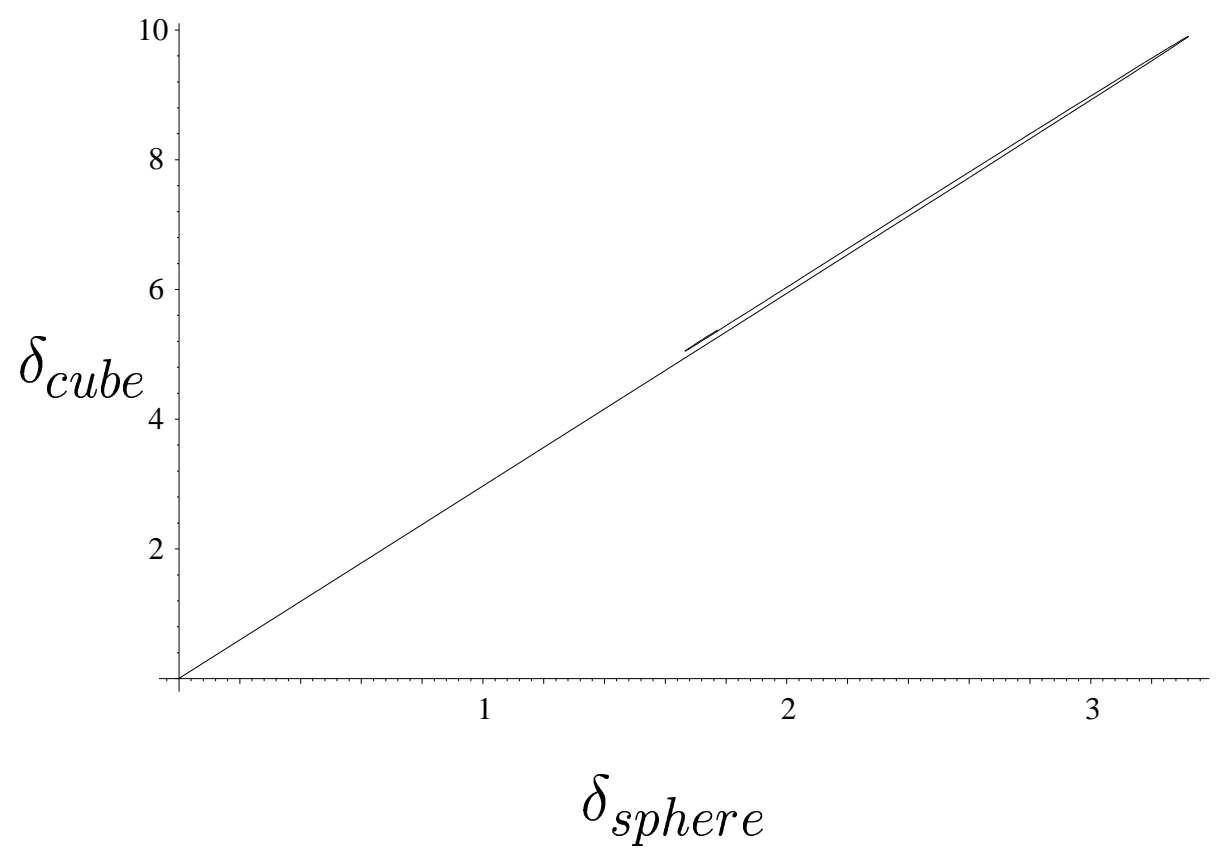

Figure 7: Comparison of the parameter values in the cube and the sphere, parameterised by the maximum temperature. 


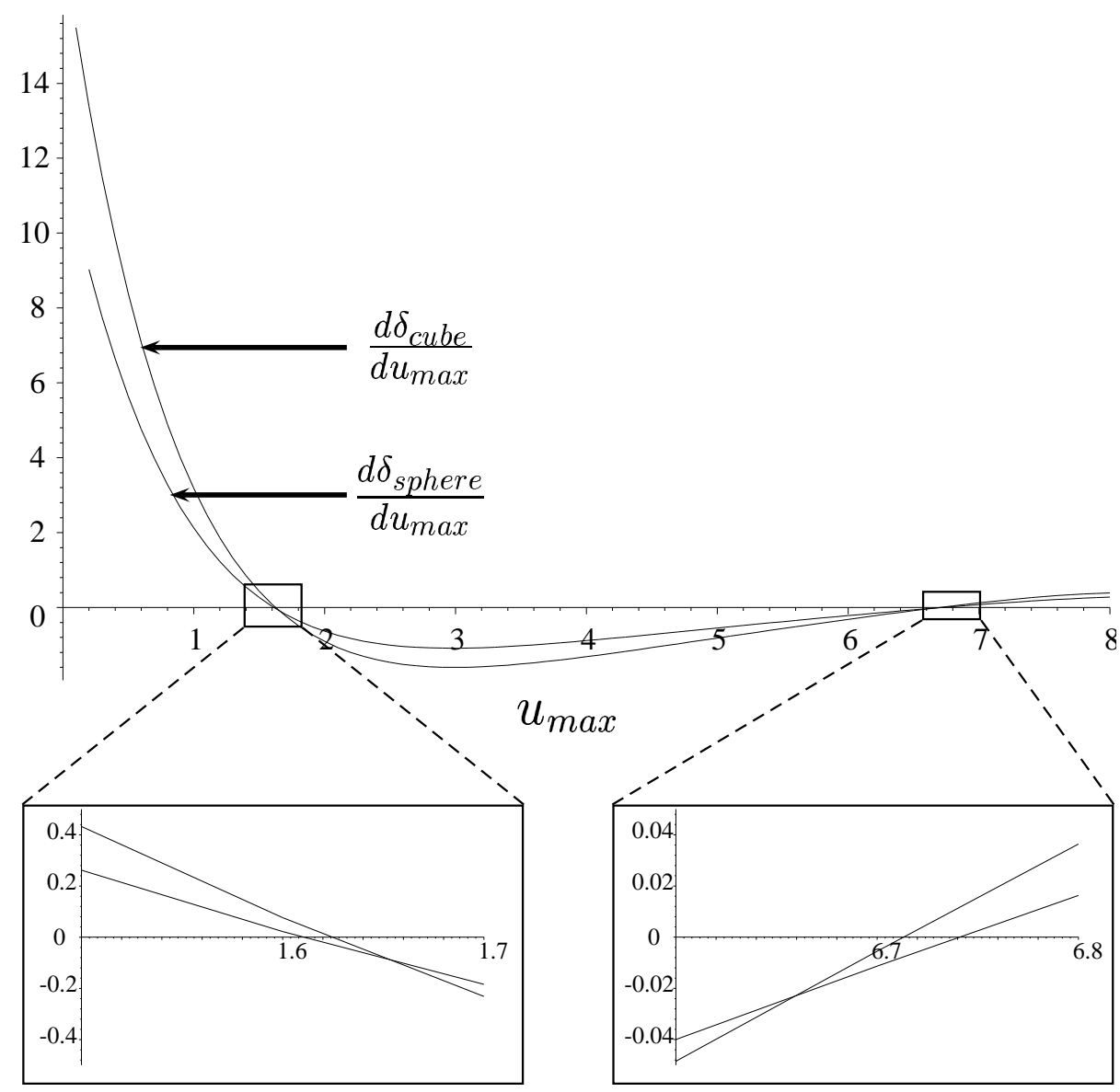

Figure 8: The derivative of the parameter values in the cube and the sphere, with respect to the maximum temperature. 


\section{Conclusions}

We have considered the thermal ignition problem in a cube. Using various numerical methods, we have been able to find regions of parameter space where there are multiple solutions, albeit unstable ones, for the steady-state problem. This behaviour has been well studied in the sphere and we have been able to find an almost linear relationship between the bifurcation diagram for the sphere and the cube. There seems every reason to expect that there will be an infinite multiplicity in the bifurcation diagram in the cube, just as is known to exist in the sphere.

\section{References}

[1] E. Balakrishnan, A. Swift and G.C. Wake "Multiple solutions in hollow geometries in the theory of thermal ignition", Appl. Math. Lett. 10 (5) pp. 41-46, 1997. C821

[2] D.A. Frank-Kamenetskii "Diffusion and heat transfer in chemical kinetics", 2nd ed. (Plenum Press, New York). 1969. C821, C824

[3] W.H. Press, S.A. Teukolsky, W.T. Vetterling, B.P. Flannery "Numerical recipes in Fortran 77 : The art of scientific computing", (Press Syndicate, University of Cambridge), pp. 854-862, 1992. C824, C825

[4] G.C. Wake and M.J. Hood "Multiplicity of solutions of a quasilinear elliptic equation in spherical domains", Mathl. Comput. Modeling 18 (10) pp. 157-162, 1993. C821 
[5] R.O. Weber, G.C. Wake, H.S. Sidhu, G.N. Mercer, B.F. Gray, W. Derrick and E. Balakrishnan "On the crossing of intermediate unstable steady state solutions for thermal ignition in a sphere", ANZIAM J. 43 pp. 77-85, 2001. C821, C822 